University of Nebraska - Lincoln

DigitalCommons@University of Nebraska - Lincoln

$1-1-2002$

\title{
Multipole Expansions of Irreducible Tensor Sets and Some Applications
}

\author{
N. L. Manakov \\ Voronezh State University, manakov@phys.vsu.ru
}

A. V. Meremianin

Voronezh State University, meremianin@phys.vsu.ru

Anthony F. Starace

University of Nebraska-Lincoln, astarace1@unl.edu

Follow this and additional works at: https://digitalcommons.unl.edu/physicsstarace

Part of the Physics Commons

Manakov, N. L.; Meremianin, A. V.; and Starace, Anthony F., "Multipole Expansions of Irreducible Tensor Sets and Some Applications" (2002). Anthony F. Starace Publications. 142.

https://digitalcommons.unl.edu/physicsstarace/142

This Article is brought to you for free and open access by the Research Papers in Physics and Astronomy at DigitalCommons@University of Nebraska - Lincoln. It has been accepted for inclusion in Anthony F. Starace Publications by an authorized administrator of DigitalCommons@University of Nebraska - Lincoln. 
Published in Journal of Physics B: Atomic, Molecular and Optical Physics 35 (2002), pp. 77-91. Online at http://stacks.iop.org/JPhysB/35/77 Copyright $\odot 2002$ Institute of Physics Publishing Ltd. Used by permission.

\title{
Multipole Expansions of Irreducible Tensor Sets and Some Applications
}

\author{
N. L. Manakov, ${ }^{1}$ A. V. Meremianin, ${ }^{1}$ and Anthony F. Starace ${ }^{2}$ \\ ${ }^{1}$ Research and Education Center, Voronezh State University, 394693 Voronezh, Russia \\ 2 Department of Physics and Astronomy, University of Nebraska-Lincoln, Lincoln, NE \\ 68588-0111, USA
}

\begin{abstract}
A formal multipole expansion of tensor functions dependent on several vector parameters is obtained in an invariant differential form. We apply this result to derive multipole expansions of finite rotation matrices in terms of finite sums of bipolar harmonics. The multipole expansion in terms of bipolar harmonics of unit vectors $\hat{r}_{1}$ and $\hat{r}_{2}$ are analyzed for functions of the type $f(r) Y_{l m}(\hat{r})$ (with $\boldsymbol{r}=\boldsymbol{r}_{1}-\boldsymbol{r}_{2}$ ), which are important in two-center problems. As another example of the application of the multipole expansion technique, reduction formulae are obtained for tensor constructions which appear in analyses of angular distributions in photoprocesses which take exact account of non-dipole (or retardation) effects. As a result, the "photon factors" in the angular distribution for an arbitrary photoprocess are expressed in an invariant form involving only the photon polarization vector and spherical harmonics of the photon wavevector.
\end{abstract}

\section{Introduction}

Separation of kinematical (i.e. dependent on the geometry, polarization states, and momentum directions of the target and projectiles) and dynamical (i.e. dependent on the energy and other quantum numbers of interacting particles) factors is an important task for analyzing many physical processes. Different methods may be used for these purposes depending on the specific character of the problem. For instance, the techniques of helicity scattering amplitudes [1] and invariant amplitudes [2] are useful for the general analysis of $S$-matrix elements in quantum electrodynamics. Such methods provide a powerful tool for the analysis of general properties of a physical phenomenon based only on symmetry considerations, taking into account the invariant (e.g. vector or spinor) characteristics inherent to the concrete problem.

In atomic physics, spherical symmetry arguments are most important both for analyzing the atomic response to external perturbations and for the separation of kinematical and dynamical factors in amplitudes and/or cross sections of atomic collisions with photons and/or electrons. Although the widely used techniques of angular momentum algebra pro- 
vide the possibility for such analysis, in general the direct use of these techniques requires tedious routine calculations of sums of Clebsch-Gordan coefficients and leads to complicated final expressions for the cross sections (involving spherical harmonics or their tensor products, i.e. multipolar harmonics of vectors of the problem). The key issue is to find a more convenient parametrization of a cross section, preferably as a combination of simple vector constructions, such as the scalar products of vectors of the problem and/or expansions in Legendre polynomials of angles between these vectors.

In a set of papers ([3-6]; see also the mathematical appendix in [7]), we have developed a number of methods for the reduction of complicated tensor constructions which appear in the theory of angular distributions involving polarized particles. In its most general formulation, our approach is based on an invariant (i.e. independent of a concrete coordinate frame) analysis of the irreducible tensor operators of rank $j, T_{j m}$, dependent on a number of vector parameters, $T_{j m}=T_{j m}\left(\boldsymbol{a}_{1}, \boldsymbol{a}_{2}, \ldots\right)$. In physical applications, the vectors $\boldsymbol{a}_{\boldsymbol{i}}$ may be associated with momenta of incident and scattered particles, spins and angular momenta of targets and projectiles etc. Irreducible tensor sets are fundamental objects of the theory of angular momentum [8] and they occur in fact (explicitly or implicitly) in any problem possessing spherical symmetry, either directly in the initial formulation (as, e.g., in the state multipoles $\rho_{j m}$ when dealing with partially polarized targets and/or projectiles) or indirectly as when they are composed of vector or spinor parameters of the problem being analyzed with the use of angular momentum algebra (as, e.g., in multipolar harmonics). The idea of an invariant parametrization of tensor operators was realized by Manakov et al. [4], where invariant representations of finite rotation matrices (FRMs), $R_{k m}^{j}(\Omega)$, were introduced (i.e. having explicit tensor forms; see section 4 below for further details). FRMs are basic objects in the quantum theory of angular momentum (see, e.g., [9]) that determine the transformation of tensor operators under the rotation $\Omega$ of a coordinate frame

$$
\tilde{T}_{j m}=\sum_{m^{\prime}=-j}^{j} T_{j m^{\prime}} R_{m^{\prime} m}^{j}(\Omega),
$$

where $T_{j m}$ and $\tilde{T}_{j m}$ are the components of an irreducible tensor $T_{j}$, given in the "old" (space-fixed) frame $K$ and in the "new" (rotated) frame $\tilde{K}$, respectively; and where $\Omega$ denotes three rotation parameters, for example the Euler angles $\alpha, \beta, \gamma$. Using the transformation rule (1), $T_{j m}$ (or more exactly, $\tilde{T}_{j m}$, i.e. the operator $T_{j m}$ in an arbitrary reference frame $\tilde{K}$ ) may be presented in terms of its $j m^{\prime}$-components in a suitable frame $K$, and of invariant FRMs, without an explicit parametrization of the rotation $\Omega$; see for instance equations (53) and (54) in [4]. Moreover, the parameters which implicitly describe the rotation $\Omega$ (e.g. two non-collinear vectors $\boldsymbol{n}$ and $\boldsymbol{n}^{\prime}$, for the case of integer $j$ [4]) may be connected with some observable vector quantities $\boldsymbol{a}_{1}, \boldsymbol{a}_{2}, \ldots$ of the problem being analyzed. Thus, the tensors $T_{j m}$, which enter the final results for cross sections after the direct use of angular momentum algebra techniques, may be presented in simplest form, directly in terms of physical observables $\boldsymbol{a}_{1}, \boldsymbol{a}_{2}, \ldots$ The invariant parametrization of the transformation rule (1) is not the only way to present complex tensor constructions in a simple invariant form (although it is the most general way). Other ways include the so-called algebraic method, which is based on the evident invariance of tensor identities to the particular choice of coordinate frame, and the reduction technique for the tensor products of spherical harmonics. This reduction technique, developed originally by Manakov et al [3] for the simplification of bipolar harmonics with higher-rank internal tensors, i.e. tensor products of spherical functions $T_{j m}\left(\boldsymbol{n}, \boldsymbol{n}^{\prime}\right) \equiv\left\{Y_{l}(\boldsymbol{n}) \otimes Y_{l^{\prime}}\left(\boldsymbol{n}^{\prime}\right)\right\}_{j m}$ with $\left(l+l^{\prime}\right)>j$, includes also an original technique of invariant vector differentiation of functions dependent on (in general, several) vector arguments. The utility of these methods for concrete physical problems was demonstrated, in particular, in papers $[3,4,7]$ by the derivation of conve- 
nient, canonical parametrizations of angular distributions for a number of photoprocesses in the electric dipole approximation (which is applicable for optical photons). Further applications have been demonstrated recently in the theory of coherent population trapping and laser cooling of atomic gases $[10,11]$ and to account for relativistic effects in twophoton transitions [12].

In this paper we present another method (i.e. in addition to those discussed in the previous paragraph) for analyzing an irreducible tensor set $T_{j m}=T_{j m}\left(\boldsymbol{a}_{1}, \boldsymbol{a}_{2}, \ldots\right)$ that is dependent on a number of vectors. Namely, the ideas of the invariant "vector differentiation technique" [3] are extended here to derive the multipole expansion of $T_{j m}$, i.e. the expansion of a general tensor $T_{j m}\left(\boldsymbol{a}_{1}, \boldsymbol{a}_{2}, \ldots\right)$ in terms of the complete set of multipolar harmonics of rank $j$ that depend only on the directions ("angular variables") of the vectors $\boldsymbol{a}_{1}, \boldsymbol{a}_{2}$, .... The multipolar (in particular, bipolar and tripolar) harmonics form a well known basis set for the multipole expansion of functions dependent on a few vector directions [9]. Such expansions are standard tools in physics, especially in quantum many-body theory and in electromagnetic theory. The regular method for calculations of the coefficients in multipole expansions is the projection technique, based on the orthogonality of harmonics with different ranks. However, the projection method allows one to obtain analytic results only for a limited set of functions having simple functional forms so that necessary integrals can be calculated analytically. We present here an alternative, differential approach, which generalizes earlier results by Sack [13] (who dealt with multipole expansions for the special case of two-center functions) as well as by Avery [14] and Wen and Avery [15] (who dealt with bicenter expansions using the vector differentiation technique in three dimensions [14] and $m$ dimensions [15] for the special case of scalar functions). Our general results for the multipole expansion of $T_{j m}\left(\boldsymbol{a}_{1}, \boldsymbol{a}_{2}, \ldots\right)$ in an invariant differential form are presented in Section 2 (cf. equations (8), (11)). This invariant differential method is flexible enough to be applied to a wide class of tensor functions $T_{k m}$. The details of the application of the formal techniques presented in Section 2 to concrete calculations are illustrated in Section 3. In that section we treat the multipole expansion (in terms of bipolar harmonics) of a tensor function that is dependent on the distance between two points specified by the vectors $\boldsymbol{r}_{1}$ and $\boldsymbol{r}_{2}$. This type of function is important in many two-center problems, and its multipole expansion, which is similar to that in our equations (17) and (18), was obtained earlier [13] by a noninvariant differential method which requires more cumbersome calculations. In section 4 we derive the multipole expansion (25) for our invariant representation for FRMs in vector form. For Euler's parametrization of the rotation $\Omega$, this surprisingly simple result (25) reduces to the multipole expansion of the Wigner $D$ functions and, further, to the compact expansion of spherical harmonics $Y_{l m}(\theta, \varphi)$ in terms of the Legendre polynomials $P_{k}(\cos \theta)$.

In section 5 we present the simplification of the tensor $T_{k m}$ which involves the tensor product of more general objects than $Y_{l m}(\boldsymbol{n})$, vector spherical harmonics $\boldsymbol{Y}_{J M}^{l}(\boldsymbol{n})$ (or electric and magnetic multipoles, $\left.\boldsymbol{Y}_{J M}^{(\lambda)}(\boldsymbol{n})\right)$, which are fundamental objects of electromagnetic theory [16] and which appear, in particular, in analyses of photon-atom interactions that go beyond the electric dipole approximation. As an example of the physical application of this result, in Section 5 we analyze angular distributions in photoprocesses and obtain the simplest invariant form for the "photon factors" of partial cross sections for an arbitrary photoprocess, taking exact account of non-dipole (retardation) effects. We present two parametrizations for "photon factors," (36) and (38), based on an extension of our previous results on the reduction of tensor products involving spherical harmonics [3] and on the use of the regular method of multipole expansions presented in section 2. As discussed in Section 5.1, these parametrizations may prove useful for the analysis of experimental data in the VUV and x-ray frequency ranges characteristic of the new generation of synchrotron light sources. 
Finally, in the appendix we present the generalization of the well known "gradient formulae" [9] for the action of a single gradient-operator on a function of the form $f(r) Y_{l m}(\hat{r})$ to the case of tensor products of several gradient-operators.

\section{Differential formulae for multipole expansions of tensor operators}

Below we present the general method for the derivation of the multipole expansions of tensor operators of rank $j$ based on the use of the differential technique, which is similar to that used in the usual Taylor expansion of some tensor function $T_{j m}(r)$ :

$$
T_{j m}(\boldsymbol{r})=\left.\sum_{n=0}^{\infty} \frac{1}{n !}\left(\boldsymbol{r} \cdot \nabla^{\prime}\right)^{n} T_{j m}\left(\boldsymbol{r}^{\prime}\right)\right|_{r^{\prime}=0},
$$

where $\nabla^{\prime}=\partial / \partial \boldsymbol{r}^{\prime}$. It is seen that different terms in this identity are homogeneous polynomials in coordinates of the vector $\boldsymbol{r}=(x, y, z)$, which can be decomposed into irreducible parts, thus establishing the dependence of the function $T_{j m}(\boldsymbol{r})$ on the coordinates of the unit vector $r / r$ in terms of infinite sums of spherical harmonics. Therefore, these sums (called "multipole expansions" of the function $T_{j m}(\boldsymbol{r})$ ) are none other than somewhat rearranged Taylor series, and our idea is to modify equation (2) in order to present the angular dependence of $T_{j m}(\boldsymbol{r})$ in the form of irreducible tensor products.

Firstly, we note that equation (2) may also be written in symbolic form as

$$
T_{j m}(\boldsymbol{r})=\left.\mathrm{e}^{r \cdot \nabla^{\prime}} T_{j m}\left(\boldsymbol{r}^{\prime}\right)\right|_{\boldsymbol{r}^{\prime}=0},
$$

where for the operator exponent on the rhs of this equation we use the standard multipole expansion of an exponential function [9]:

$$
\mathrm{e}^{r \cdot a}=\sum_{l=0}^{\infty} \frac{(2 l+1) ! !}{l !}\left(\{\boldsymbol{r}\}_{l} \cdot\{\boldsymbol{a}\}_{l}\right)(r a)^{-l} j_{l}(r a) .
$$

The notation $\{\boldsymbol{r}\}_{l}$ is used for the tensor product of $l$ vectors $\boldsymbol{r}$, which is related to the spherical harmonic $[3,9]$ as follows:

$$
\{r\}_{l m} \equiv\left\{\ldots\left\{\boldsymbol{r} \otimes\{\boldsymbol{r} \otimes r\}_{2}\right\}_{3} \ldots \otimes r\right\}_{l m}=\sqrt{\frac{4 \pi l !}{(2 l+1) ! !}} r^{l} Y_{l m}(\hat{r}), \quad \hat{r}=r / r .
$$

(Note that the multipole expansion of the finite rotation operator, $\exp (-\mathrm{i} \omega \boldsymbol{n} \cdot \boldsymbol{j})$, in a (finite) sum of spherical harmonics similar to the expansion (4) was used by Happer [17].) The spherical Bessel function, $j_{l}(r a)$, in (4) is connected with the hypergeometric function ${ }_{0} F_{1}(a ; x)[18]:$

$$
(r a)^{-l} j_{l}(r a)=\frac{1}{(2 l+1) ! !}{ }_{0} F_{1}\left(l+\frac{3}{2} ;-\frac{1}{4} r^{2} a^{2}\right) .
$$

Taking account of this equation and noting that in our case $\boldsymbol{a}=\nabla^{\prime}$, equation (3) for $T_{j m}(\boldsymbol{r})$ may be rewritten as

$$
T_{j m}(\boldsymbol{r})=\left.\sum_{l=0}^{\infty} \frac{1}{l !}{ }_{0} F_{1}\left(l+\frac{3}{2} ;-\frac{1}{4} r^{2} \nabla^{\prime 2}\right)\left(\{\boldsymbol{r}\}_{l} \cdot\left\{\nabla^{\prime}\right\}_{l}\right) T_{j m}\left(\boldsymbol{r}^{\prime}\right)\right|_{r^{\prime}=0} .
$$

The use of the tensor recoupling rule [9] leads to the following identity:

$$
\left(\{r\}_{l} \cdot\left\{\nabla^{\prime}\right\}_{l}\right) T_{j m}\left(r^{\prime}\right)=\sum_{l^{\prime}=|l-j|}^{l+j}(-1)^{j-l^{\prime}} \sqrt{\frac{2 l^{\prime}+1}{2 j+1}}\left\{\{r\}_{l} \otimes\left\{\left\{\nabla^{\prime}\right\}_{l} \otimes T_{j}\left(r^{\prime}\right)\right\}_{l^{\prime}}\right\}_{j m} .
$$


Inserting (7) into (6) we obtain finally

$$
\begin{aligned}
& T_{j m}(\boldsymbol{r})=\sum_{l=0}^{\infty} \frac{1}{l !}{ }_{0} F_{1}\left(l+\frac{3}{2} ;-\frac{1}{4} r^{2} \nabla^{\prime 2}\right) \\
& \times\left.\sum_{l^{\prime}=|l-j|}^{l+j}(-1)^{j-l^{\prime}} \sqrt{\frac{2 l^{\prime}+1}{2 j+1}}\left\{\{r\}_{l} \otimes\left\{\left\{\nabla^{\prime}\right\}_{l} \otimes T_{j}\left(\boldsymbol{r}^{\prime}\right)\right\}_{l^{\prime}}\right\}_{j m}\right|_{r^{\prime}=0} .
\end{aligned}
$$

Obviously, the entire dependence of the rhs of this equation on the direction of the vector $\boldsymbol{r}$ is given by the term $\{\boldsymbol{r}\}_{l}$, which is in fact the spherical harmonic (5). Thus, equation (8) gives the most general, symbolic form for the multipole expansion of the tensor $T_{j m}(r)$ into the (generally infinite) sum of spherical harmonics of $\hat{r}$. The calculation of coefficients in the multipole expansion (8), i.e. of derivatives of $T_{j m}(\boldsymbol{r})$ at $\boldsymbol{r}=0$, may be performed using methods for invariant vector differentiation (see [3]). If the tensor $T_{j m}$ depends (besides $\boldsymbol{r}$ ) on other vectors, $\boldsymbol{a}_{1}, \boldsymbol{a}_{2}, \ldots$, as the result of such differentiation the spherical harmonics of vectors $\boldsymbol{a}_{\boldsymbol{i}}$ will enter the final result. Thus, in general, equation (8) is a multipole expansion in a series of multipolar harmonics of rank $j$.

If the tensor $T_{j m}$ depends on only two vectors, $\boldsymbol{r}$ and $\boldsymbol{a}$, then the general form of the coefficients is

$$
\left.{ }_{0} F_{1}\left(l+\frac{3}{2} ;-\frac{1}{4} r^{2} \nabla^{\prime 2}\right)\left\{\left\{\nabla^{\prime}\right\}_{l} \otimes T_{j}\left(\boldsymbol{r}^{\prime}, \boldsymbol{a}\right)\right\}_{l^{\prime} m^{\prime}}\right|_{r^{\prime}=0}=C_{l l^{\prime}}(r) \tilde{Y}_{l^{\prime} m^{\prime}}(\boldsymbol{a}),
$$

where, for simplicity, we suppose that $|\boldsymbol{a}|=1$, and where $\tilde{Y}_{l m}(\boldsymbol{a})$ are "renormalized" spherical harmonics, $\tilde{Y}_{l m}(\boldsymbol{a}) \equiv(4 \pi /(2 l+1))^{1 / 2} Y_{l m}(\boldsymbol{a})$. Indeed, the lhs of (9) does not depend on the vector $\boldsymbol{r}^{\prime}$ (after the differentiation and evaluation of the result at $\boldsymbol{r}^{\prime}=0$ ) while it is a tensor of rank $l^{\prime}$. Hence, it is proportional to the spherical harmonic of the unit vector $\boldsymbol{a}$, which is the only vector quantity in the problem. The scalar factor $C_{l l^{\prime}}(r)$ in (9) may be calculated in any suitable coordinate frame, for example in the frame $K$ whose $z$-axis is directed along the vector $\boldsymbol{a}$. In this frame, only the component $a_{0}=a_{z}=1$ is nonzero, and thus equation (9) gives

$$
C_{l l^{\prime}}(r)=\left.{ }_{0} F_{1}\left(l+\frac{3}{2} ;-\frac{1}{4} r^{2} \nabla^{\prime 2}\right) \sum_{n} C_{l n j-n}^{l^{\prime} 0}\left\{\nabla^{\prime}\right\}_{l n} T_{j,-n}\left(\boldsymbol{r}^{\prime}, \boldsymbol{a}\right)\right|_{r^{\prime}=0} .
$$

This result shows, in particular, that the calculation of $C l l^{\prime}$ simplifies drastically if $T_{j m}(\boldsymbol{r})$ obeys the Laplace equation, $\nabla^{2} T_{j m}(\boldsymbol{r})=0$. Hence, for this case the series expansion for the function ${ }_{0} F_{1}$ in (10) may be replaced by unity. Equations (8) and (9) lead to the following final result for the multipole expansion of a tensor function of two independent vectors, $\boldsymbol{r}$ and $\boldsymbol{a}$ :

$$
T_{j m}(\boldsymbol{r}, \boldsymbol{a})=\frac{4 \pi}{\sqrt{2 j+1}} \sum_{l=0}^{\infty} \sqrt{\frac{2^{l}}{(2 l+1) !}} \sum_{l^{\prime}=|l-j|}^{l+j}(-1)^{j-l^{\prime}} C_{l l^{\prime}}(r) r^{l}\left\{Y_{l}(\hat{r}) \otimes Y_{l^{\prime}}(\boldsymbol{a})\right\}_{j m} .
$$

Obviously, this is an expansion in a complete set of bipolar harmonics.

\section{Multipole expansion of two-center functions of the form $f(r) Y_{j m}(\hat{r})$}

As an illustration of the application of the general formulae derived in section 2, we consider first the expansion in bipolar harmonics (of arguments $\hat{r}_{1}$ and $\hat{r}_{2}$ ) of a tensor function $T_{j m}\left(\boldsymbol{r}_{1}, \boldsymbol{r}_{2}\right)$ that is dependent only on the direction and magnitude of the vector argument $\boldsymbol{r}=\boldsymbol{r}_{1}-\boldsymbol{r}_{2}$ :

$$
T_{j m}\left(\boldsymbol{r}_{1}, \boldsymbol{r}_{2}\right)=f(r) Y_{j m}(\hat{r}),
$$

where $f(r)$ is an arbitrary (differentiable) function of $r=\left|\boldsymbol{r}_{1}-\boldsymbol{r}_{2}\right|$. Such functions of the distance between two points specified by the vectors $\boldsymbol{r}_{1}$ and $\boldsymbol{r}_{2}$ are important in many twocenter problems. A number of results for concrete choices of $f(r)$ are presented in [9], and 
an analysis of the general form of $f(r)$ was performed by Sack [13]. Below we shall show that for the case considered here the expressions for the coefficients in the general multipole expansion (8) may be reduced to a simpler form, not involving gradient-operators.

In order to obtain explicit expressions for the coefficients of the multipole expansion of the function (12), we shall need to calculate tensor products involving gradient-operators that appear in two equivalent forms,

$$
\left.\left\{\left\{\nabla^{2}\right\}_{l} \otimes f(r) \tilde{Y}_{j}(\hat{r})\right\}_{l^{\prime} m^{\prime}}\right|_{r_{2}=0}=(-1)^{l}\left\{\left\{\nabla_{1}\right\}_{l} \otimes f\left(r_{1}\right) \tilde{Y}_{j}\left(\hat{r}_{1}\right)\right\}_{l^{\prime} m^{\prime}},
$$

where $\nabla_{i}=\partial / \partial \boldsymbol{r}_{i}$. For $l=1$, the differentiation in the tensor product on the rhs of (13) can be performed using the well known "gradient formulae" (see, e.g., ch. 5, section 5.8.3 in [9]) for the action of a single gradient-operator on a function of the form $f(r) Y_{l m}(\hat{r})$. The generalization of these formulae to the case of tensor products of several gradient-operators is given in the appendix. This more general result is

$$
\left\{\{\nabla\}_{l} \otimes f(r) \tilde{Y}_{j}(\hat{r})\right\}_{l^{\prime} m^{\prime}}=\sqrt{\frac{l !}{(2 l-1) ! !}} C_{l 0 j 0}^{l^{\prime} 0} \frac{1}{r^{l^{\prime}+1}} \tilde{Y}_{l^{\prime} m^{\prime}}(\hat{r}) \hat{\mathrm{d}}^{g-l^{\prime}} r^{2 g+1} \hat{\mathrm{d}}^{g-j} \frac{f(r)}{r^{j}},
$$

where $\hat{\mathrm{d}}=(1 / r)(\mathrm{d} / \mathrm{d} r)$ and where $g \equiv 1 / 2\left(l+l^{\prime}+j\right)$ is an integer number since $\left(l+l^{\prime}+j\right)$ is even because of the properties of the Clebsch-Gordan coefficients $C_{l 0 j 0}^{l^{\prime} 0}$. Note that, depending on the explicit form of the function $f(r)$ in (12), it may be convenient to use an alternative form of equation (14), given by (A.7), involving another ordering of the derivative operators $\hat{d}$.

Taking into account (14), the general equation (8) for the function (12) may be written (after some rearrangements of summation indices) as

$$
\begin{aligned}
f(r) Y_{j m}(\hat{r})= & \sum_{l^{\prime}=0}^{\infty} \frac{r_{2}^{l^{\prime}}}{\left(2 l^{\prime}-1\right) ! !}{ }_{0} F_{1}\left(l^{\prime}+\frac{3}{2} ;-\frac{1}{4} r_{2}^{2} \nabla_{1}^{2}\right) \sum_{l=\left|l^{\prime}-j\right|}^{l^{\prime}+j} C_{l^{\prime} 0 j 0}^{l 0}\left[\hat{\mathcal{D}}_{l l^{\prime}}^{(j)}\left(r_{1}\right) f\left(r_{1}\right)\right] \\
& \times \frac{\sqrt{(2 l+1)}}{2 j+1}\left\{\tilde{Y}_{l}\left(\hat{r}_{1}\right) \otimes \tilde{Y}_{l^{\prime}}\left(\hat{r}_{2}\right)\right\}_{j m},
\end{aligned}
$$

where the differential operator $\hat{\mathcal{D}}$ is defined by

$$
\hat{\mathcal{D}}_{l l^{\prime}}^{(j)}\left(r_{1}\right)=r_{1}^{l}\left(\frac{1}{r_{1}} \frac{\mathrm{d}}{\mathrm{d} r_{1}}\right)^{\left(l+l^{\prime}-j\right) / 2} \frac{1}{r_{1}^{l-l^{\prime}+j+1}}\left(\frac{1}{r_{1}} \frac{\mathrm{d}}{\mathrm{d} r_{1}}\right)^{\left(l^{\prime}-l+j\right) / 2} r_{1}^{j+1} .
$$

Taking into account the known identity

$$
\nabla^{2} \varphi(r) Y_{l m}(\hat{r})=Y_{l m}(\hat{r})\left(\frac{1}{r^{2}} \frac{\mathrm{d}}{\mathrm{d} r} r^{2} \frac{\mathrm{d}}{\mathrm{d} r}-\frac{l(l+1)}{r^{2}}\right) \varphi(r),
$$

we obtain the multipole expansion for the function (12) in the following final form:

$$
f(r) Y_{j m}(\hat{r})=\sum_{l=0}^{\infty} \sum_{l^{\prime}=|l-j|}^{l+j} \sqrt{4 \pi\left(2 l^{\prime}+1\right)} C_{j 0 l^{\prime} 0}^{l 0} B_{l l^{\prime}}^{(j)}\left(r_{1}, r_{2}\right)\left\{Y_{l}\left(\hat{r}_{1}\right) \otimes Y_{l^{\prime}}\left(\hat{r}_{2}\right)\right\}_{j m},
$$

where the coefficients $B$ are defined by

$$
\begin{aligned}
B_{l l^{\prime}}^{(j)}\left(r_{1}, r_{2}\right)= & \frac{r_{2}^{l^{\prime}}}{\left(2 l^{\prime}+1\right) ! !} \sum_{k=0}^{\infty} \frac{(-1)^{k}}{k !\left(l^{\prime}+3 / 2\right)_{k}}\left(\frac{r_{2}}{2}\right)^{2 k} \\
& \times\left[\frac{1}{r_{1}^{2}} \frac{\mathrm{d}}{\mathrm{d} r_{1}} r_{1}^{2} \frac{\mathrm{d}}{\mathrm{d} r_{1}}-\frac{l(l+1)}{r_{1}^{2}}\right]^{k} \hat{\mathcal{D}}_{l l^{\prime}}^{(j)}\left(r_{1}\right) f\left(r_{1}\right) .
\end{aligned}
$$

Note that the $\hat{\mathcal{D}}$-operator (16) commutes with the $\nabla^{2}$-operator because $\hat{\mathcal{D}}$ originates from $\nabla$-operators (see (8) and (A.2)). Thus, we can write the operator $\hat{\mathcal{D}}_{l l^{\prime}}^{(j)}\left(r_{1}\right)$ in (18) before the sum over the index $k$. 
The results for an arbitrary $f(r)$ given by equations (17), (18) are closely related to the results of Sack in [13], where they were derived for the first time based on the multipole expansion for the function $r^{n} Y_{j m}(\hat{r})$, with $\boldsymbol{r}=\boldsymbol{r}_{1}-\boldsymbol{r}_{2}$. Unlike the results in [13], our operator expression in (18) for the coefficients $B$ does not depend on the relation between the indices $l, l^{\prime}$ and $j$. In addition, by dealing initially with an arbitrary $f(r)$, we have obtained the nontrivial relation (A.8) for the differential operators $\hat{\mathrm{d}}=(1 / r)(\mathrm{d} / \mathrm{d} r)$. For the special choice of the function $f(r), f(r)=r^{n}$, the explicit form of the coefficients in the multipole expansion (17) may be easily derived from (18),

$$
\begin{aligned}
B_{l l^{\prime}}^{(j)}\left(r_{1}, r_{2}\right)= & \frac{2^{l^{\prime}}}{\left(2 l^{\prime}+1\right) ! !}\left(\frac{j-n}{2}\right)_{\left(l^{\prime}+l-j\right) / 2}\left(-\frac{j+n+1}{2}\right)_{\left(l^{\prime}-l+j\right) / 2} r_{1}^{n}\left(-\frac{r_{2}}{r_{1}}\right)^{l^{\prime}} \\
& \times{ }_{2} F_{1}\left(\frac{l^{\prime}-l-n-1}{2}, \frac{l+l^{\prime}-n}{2} ; l^{\prime}+\frac{3}{2} ; \frac{r_{2}^{2}}{r_{1}^{2}}\right),
\end{aligned}
$$

where ${ }_{2} F_{1}(\alpha, \beta ; \gamma ; x)$ is the Gauss hypergeometric function. These results coincide with those derived by Sack [13]. As we mentioned above in Section 2, the multipole expansion simplifies for tensor functions $F_{j m}(\boldsymbol{r})$ obeying the Laplace equation, $\nabla^{2} F_{j m}(\boldsymbol{r})=0$. Namely, for $f(r)=r^{j}$ (or $f(r)=r^{-j-1}$ ) the Pochhammer symbols in (19) are nonzero only for $j=l+$ $l^{\prime}$ (or $j=l-l^{\prime}$ ). Therefore, the summations in (17) over $l$ and $l^{\prime}$ become finite and the hypergeometric function in (19) reduces to unity. We do not present here explicit expressions for these well known results [9].

\section{Multipole expansion of finite rotation matrices}

The FRMs $R_{k m}^{j}(\Omega)$ constitute an irreducible tensor set having rank $j$ and projections $m$. The tensor sense of the index $m$ is obvious from equation (1), which may be interpreted as the expansion of a tensor $\tilde{T}_{j m}$ (in the rotated frame) in $(2 j+1)$ tensors $R_{m^{\prime} m}^{j}(\Omega)$ enumerated by the (non-tensor) index $m^{\prime}$. Recently we introduced the so-called invariant representations of FRMs (see [4, 5] for the case of integer ranks $j$ and more general results in [6] that are valid for both integer and half-integer $j$ ), which are useful, in particular, for analyses of angular distributions in processes involving polarized particles. For the case of integer $j$, these invariant representations have the simplest form for symmetrized combinations of $R_{k m}^{j}(\Omega)$, i.e. the "parity-projected" FRMs, which are defined by [5]

$$
R_{k m}^{j \lambda_{\mathrm{p}}}(\Omega)=\left(1-\frac{\delta_{0, k}}{2}\right)\left(R_{-k m}^{j}(\Omega)+(-1)^{k+\lambda_{\mathrm{p}}} R_{k m}^{j}(\Omega)\right), \quad k \geqslant \lambda_{\mathrm{p}},
$$

where $\lambda_{\mathrm{p}}=0\left(\lambda_{\mathrm{p}}=1\right)$ for even (odd) parity. Manakov et al [4] obtained several alternative invariant forms for the FRMs $R_{k m}^{j \lambda}$. The most general result for parity-projected FRMs can be expressed in terms of bipolar harmonics depending on two vectors, $\boldsymbol{n}$ and $\boldsymbol{n}^{\prime}$, connected with the fixed frame $K$ (cf. equation (7) in [5]):

$$
R_{k m}^{j \lambda_{\mathrm{p}}}(\Omega)=\sum_{s=0}^{k-\lambda_{\mathrm{p}}} A_{k s}^{\lambda_{\mathrm{p}}}(\theta)\left\{Y_{j-s}(\boldsymbol{n}) \otimes Y_{s+\lambda_{\mathrm{p}}}\left(\boldsymbol{n}^{\prime}\right)\right\}_{j m},
$$

where the coefficients $A^{\lambda_{\mathrm{p}}}(\theta)$ depend on the angle $\theta$ between the vectors $\boldsymbol{n}$ and $\boldsymbol{n}^{\prime}(0<\theta$ $<\pi)$ [5], and where the tensor products $\left\{Y_{j-s}(\boldsymbol{n}) \otimes Y_{s+\lambda_{\mathrm{p}}}\left(\boldsymbol{n}^{\prime}\right)\right\}_{j m}$ are the so-called "minimal bipolar harmonics." The vector $\boldsymbol{n}$ is directed along the $z$-axis of the fixed frame $K$ and the vector $\boldsymbol{n}^{\prime}$ lies in the $z x$-plane. (Thus, the angle $\theta$ is the free parameter, and the three independent real parameters of the rotation $\Omega$ in our approach are determined by the angular coordinates of $\boldsymbol{n}$ and $\boldsymbol{n}$ ' in the "new" (or rotated) frame $\tilde{K}$.) We use the term "minimal bi- 
polar harmonics" for the parity-projected tensor products $\left\{Y_{j-s}(\boldsymbol{n}) \otimes Y_{s+\lambda_{\mathrm{p}}}\left(\boldsymbol{n}^{\prime}\right)\right\}_{j m}$ (with in$\operatorname{dex} s=0,1, \ldots, j-\lambda_{\mathrm{p}}$ ) since they form the (minimal) basis set of $(2 j+1)$ irreducible tensors in a space of tensors with an integer rank $j$. (There are $(j+1)$ polar tensors for $\lambda_{\mathrm{p}}=0$ and $j$ axial tensors (pseudotensors) for $\lambda_{\mathrm{p}}=1$.)

Obviously the representation of the FRM in equation (21) is not actually a multipole expansion since the coefficients $A_{k s}^{\lambda_{\mathrm{p}}}(\theta)$ are $\theta$ dependent. (They are in fact proportional to the Gegenbauer polynomials $C_{\substack{1 / 2-k \\ k-s-\lambda_{\mathrm{p}}}}(\cos \theta)$; see $[4,5]$ for details.) Since the FRM in an invariant vector form may be considered as a special case of a tensor function $T_{j m}$ composed of two unit vectors, $\boldsymbol{n}$ and $\boldsymbol{n}^{\prime}$, the general result (11) allows one to obtain the actual multipole expansion of our invariant expressions for the FRM, i.e. the expansion of $R_{k m}^{j}\left(\boldsymbol{n}, \boldsymbol{n}^{\prime}\right)$ in terms of regular (not "minimal") bipolar harmonics of vectors $\boldsymbol{n}$ and $\boldsymbol{n}$ ' with angle-independent numerical coefficients. For this derivation we use the following representation of FRM (see equation (17) in [4]):

$$
R_{ \pm k m}^{j}(\Omega)=A_{j k}\left\{\left\{e_{\mp 1}\right\}_{k} \otimes\left\{e_{0}\right\}_{j-k}\right\}_{j m}, \quad k \geqslant 0,
$$

where the coefficients $A_{j k}$ are

$$
A_{j k}=(-1)^{k} \sqrt{\frac{2^{k-j}(2 j) !}{(j-k) !(j+k) !}},
$$

and where $\boldsymbol{e}_{0}$ and $\boldsymbol{e}_{\mp 1}$ are spherical basis vectors of the ("old") frame $K$. For the basis vectors $\boldsymbol{e}_{0, \pm 1}$ in (22) we shall take combinations of two non-collinear unit vectors $\boldsymbol{n}$ and $\boldsymbol{n}^{\prime}$ whose orientations in the "old" frame $K$ have been discussed above, after equation (21). Thus, we have $\boldsymbol{e}_{0} \equiv \boldsymbol{n}$,

$$
e_{ \pm 1}=\left.\mp \frac{1}{\sqrt{2} \sin \theta}(\boldsymbol{r}-\boldsymbol{n}(\boldsymbol{r} \cdot \boldsymbol{n}) \pm \mathrm{i}[\boldsymbol{n} \times \boldsymbol{r}])\right|_{\boldsymbol{r}=\boldsymbol{n}^{\prime}}
$$

In terms of $\boldsymbol{n}$ and $\boldsymbol{n}^{\prime}$, equation (22) takes the form

$$
R_{ \pm k m}^{j}(\Omega)=\left.A_{j k}\left(\frac{ \pm 1}{\sqrt{2} \sin \theta}\right)^{k}\left\{\{r-n(r \cdot n) \mp \mathrm{i}[n \times r]\}_{k} \otimes\{n\}_{j-k}\right\}_{j m}\right|_{r=n^{\prime}} .
$$

In the frame $K$ the tensor product in (23) has the following form:

$$
A_{j k}\left\{\{\boldsymbol{r}-\boldsymbol{n}(\boldsymbol{r} \cdot \boldsymbol{n}) \mp \mathrm{i}[\boldsymbol{n} \times \boldsymbol{r}]\}_{k} \otimes\{\boldsymbol{n}\}_{j-k}\right\}_{j m}=\left(-2 r_{ \pm 1}\right)^{k} \delta_{m, \pm k} .
$$

It therefore satisfies the Laplace equation, thus simplifying the evaluation of (10) for the coefficients $C_{l l^{\prime}}(r)$ :

$$
\begin{aligned}
C_{l l^{\prime}} & =\sum_{n} C_{l n j-n}^{l^{\prime} 0}\{\nabla\}_{l n}\left(r_{-1}\right)^{k} \\
& =C_{k k j-k}^{\prime^{\prime} 0}\left(\nabla_{1}\right)^{k}\left(r_{-1}\right)^{k} \delta_{n, k} \delta_{l, k}=C_{k k j-k}^{l^{\prime} 0}(-1)^{k} k ! \delta_{n, k} \delta_{l, k} .
\end{aligned}
$$

Substituting the last of these equations into (11), we obtain the desired multipole expansion of the FRM:

$$
\begin{aligned}
R_{ \pm k m}^{j}(\Omega)= & \frac{4 \pi}{\sqrt{2 j+1}}\left(\frac{-1}{\sin \theta}\right)^{k} \sqrt{\frac{(2 k) ! !}{(2 k+1) ! !}} \sum_{l=j-k}^{j+k}( \pm 1)^{j-l} C_{k k j-k}^{l 0} \\
& \times\left\{Y_{k}\left(\boldsymbol{n}^{\prime}\right) \otimes Y_{l}(\boldsymbol{n})\right\}_{j m}, \quad k \geqslant 0 .
\end{aligned}
$$

This important identity can be verified straightforwardly by considering the zero rotation, $\Omega=0$. Indeed, in [4] it was noted that if an irreducible tensor of the rank $j m$ is equal to $\delta_{k, m}$ for $\Omega=0$, then (for nonzero $\Omega$ ) it coincides with the FRM $R^{j}{ }_{k m}(\Omega)$. This is a simple consequence of the invariance of tensor identities with respect to the choice of coordinate 
frame. For $\Omega=0$, the vector arguments of the spherical harmonics in (25) are $\boldsymbol{n}=(0,0)$ and $\boldsymbol{n}^{\prime}=(\theta, 0)$. Thus the bipolar harmonic on the rhs of (25) reduces to a Clebsch-Gordan coefficient and a spherical harmonic $Y_{k \pm k}(\theta)$. When the latter spherical harmonic is evaluated explicitly, all $l$-independent factors on the rhs of (25) are cancelled. Further, the sum of the product of two Clebsch-Gordan coefficients is equal to $\delta_{ \pm k, m}$ because of their orthogonality properties, and, hence, the result (25) is valid. Note that the Clebsch-Gordan coefficient on the rhs of (25) may be given in closed form without any summations [9] as

$$
C_{k k j-k}^{l 0}=\sqrt{\frac{(2 l+1)(2 k) !(-k+j+l) !(j+k) !}{(j+k+l+1) !(k-j+l) !(k+j-l) !(j-k) !}} .
$$

The multipole expansion (25) gives one additional (to that in equation (21)) invariant representation of the FRM in terms of bipolar harmonics of two independent vectors. However, these harmonics are not "minimal": the sum of the ranks of their internal spherical harmonics, $k+l$, exceeds the external one, $j$. We note the surprisingly simple form of the multipole expansion (25) which involves only one Clebsch-Gordan coefficient, (26).

As has been demonstrated in [6], for the parametrization of a rotation $\Omega$ in terms of Euler angles, invariant representations of FRMs reduce to the well known Wigner $D$-functions [9],

$$
R_{k m}^{j}(\alpha, \beta, \gamma) \equiv D_{k m}^{\dot{j}}(\alpha, \beta, \gamma)=\exp (-\mathrm{i} k \alpha) \mathrm{d}_{k m}^{j}(\beta) \exp (-\mathrm{i} m \gamma) .
$$

Thus, for Euler's parametrization of $\Omega$, the result (25) should give the multipole expansion of the Wigner $\boldsymbol{d}^{j}(\beta)$-matrix. The desired expansion may be established by considering the auxiliary rotation described by the Euler angles $(0, \beta, 0)$. This corresponds to the following choice of vectors $\boldsymbol{n}$ and $\boldsymbol{n}^{\prime}: \boldsymbol{n}=(\beta, \pi)$ and $\boldsymbol{n}^{\prime}=(0,0)$. Using this parametrization in (25) it is easy to obtain the expansion of $\boldsymbol{d}^{j}(\beta)$ in spherical harmonics $Y_{l m}(\beta, \varphi)$ with $\varphi=0$ (or in associated Legendre polynomials, $P_{l}^{|m|}(\cos \beta)$ ):

$$
\mathrm{d}_{ \pm k m}^{j}(\beta)=\sqrt{\frac{(2 k) ! !}{(2 k-1) ! !}} \frac{(-1)^{k+m}}{(\sin \beta)^{k}} \sum_{l=j-k}^{j+k}(\mp 1)^{j+l} C_{k k j-k}^{l 0} C_{k 0 j m}^{l m} \tilde{Y}_{l m}(\beta, 0) .
$$

The corresponding result for the total Wigner function $D_{k m}^{j}$ in (27) is

$$
D_{k m}^{j}(\alpha, \beta, \gamma)=\frac{(-1)^{k}}{\tilde{Y}_{|k| k}(\beta, \alpha)} \sum_{l=j-|k|}^{j+|k|} C_{|k|-k j k}^{l 0} C_{|k| 0 j m}^{l m} \tilde{Y}_{l-m}(\beta, \gamma) .
$$

Note that the expansion (28) and thus (29) can also be easily obtained straightforwardly using the addition theorem [9] for the product of $\boldsymbol{d}^{j}(\beta)$-matrices, $\mathrm{d}_{k m}^{j}(\beta) \mathrm{d}_{-k 0}^{k}(\beta)$.

For $m=0$, the $D$-function reduces to a spherical harmonic [9]. Thus, using (26) and the explicit form for $C_{k 0 j 0}^{10}$ [9], from (29) follows the expansion of the spherical harmonic $Y_{l m}(\theta, \varphi)$ in Legendre polynomials $P_{q}(\cos \theta)$ :

$$
\begin{aligned}
\tilde{Y}_{l \pm m}(\theta, \varphi)= & ( \pm 1)^{m} \sqrt{\frac{(l+m) !}{(l-m) !}} \frac{\mathrm{e}^{ \pm \mathrm{i} m \varphi}}{(\sin \theta)^{m}} \\
& \times \sum_{n=0}^{m}(-1)^{n}\left(\begin{array}{c}
n \\
m
\end{array}\right) \frac{(2 l+2 m+1-4 n)(2 l-1-2 n) ! !}{(2 l+2 m+1-2 n) ! !} P_{l+m-2 n}(\cos \theta),
\end{aligned}
$$

where $\left(\begin{array}{l}n \\ m\end{array}\right)$ is the binomial coefficient. For $m \leq 4$, results calculated according to (30) coincide with those presented in section 5.13.2 of [9]. However, we believe the compact expression (30) for arbitrary values of $m$ is a new result. 


\section{Application of multipole expansions to the case of photoprocesses involving non- dipole (retardation) effects}

In this section we shall demonstrate the utility of our invariant techniques for the reduction of tensor products involving vector spherical harmonics, $\boldsymbol{Y}_{J M}^{l}(\boldsymbol{n})$, or their linear combinations, the electric (for $\lambda=1$ ) and magnetic (for $\lambda=0$ ) multipoles, $\boldsymbol{Y}_{J M}^{(\lambda)}(\boldsymbol{n})$ [9]. These objects form a convenient basis for expansions of vector functions $\boldsymbol{F}(\boldsymbol{r})$, and they are especially useful in electromagnetic theory (see, e.g., $[2,16])$. We illustrate our method by an analysis of the general structure of the differential cross sections in quantum electrodynamical calculations of photoprocesses involving $\mathrm{x}-$ or $\gamma$-rays, when a correct accounting for non-dipole (retardation) effects in the photon-atom interaction is required.

In the radiation gauge, the operator for the electron-photon interaction is defined by the scalar product of Dirac matrices with the vector-potential, $A_{\mu}=e_{\mu} \exp (\mathrm{i} \boldsymbol{k} \cdot \boldsymbol{r})$, where $e_{\mu}$ are the spherical components of the (generally complex) polarization vector $\boldsymbol{e}$ of a photon having frequency $\omega$ and wavevector $\boldsymbol{k}=(\omega / c) \boldsymbol{n} ; \boldsymbol{e} \cdot \boldsymbol{k}=0, \boldsymbol{e} \cdot \boldsymbol{e}^{*}=1$. In order to integrate over $\boldsymbol{r}$ in calculating $S$-matrix elements for electromagnetic transitions, $\boldsymbol{A}(\boldsymbol{r})$ is expanded in vector spherical harmonics [9]:

$$
\boldsymbol{A}(\boldsymbol{r})=4 \pi \sum_{J M} \sum_{\lambda=0,1} \mathrm{i}^{J-\lambda}\left(e \cdot \boldsymbol{Y}_{J M}^{(\lambda) *}(\boldsymbol{n})\right) \boldsymbol{a}_{J M}^{(\lambda)}(\omega ; \boldsymbol{r}),
$$

where the coefficients $\boldsymbol{a}$ are defined by

$$
\begin{aligned}
& \boldsymbol{a}_{J M}^{(0)}(\omega ; \boldsymbol{r})=j_{J}(k r) \boldsymbol{Y}_{J M}^{J}(\hat{r}), \\
& \boldsymbol{a}_{J M}^{(1)}(\omega ; \boldsymbol{r})=\sqrt{\frac{J+1}{2 J+1}} j_{J-1}(k r) \boldsymbol{Y}_{J M}^{J-1}(\hat{r})-\sqrt{\frac{J}{2 J+1}} j_{J+1}(k r) \boldsymbol{Y}_{J M}^{J+1}(\hat{r}),
\end{aligned}
$$

where $j_{J}(k r)$ is a spherical Bessel function.

Since the polarization-angular and $\boldsymbol{r}$ dependences in (31) are factorized, the use of (31) leads to the following general parametrization of the cross-section of an arbitrary photoprocess:

$$
\frac{\mathrm{d} \sigma}{\mathrm{d} \Omega}=\frac{1}{4 \pi} \sum_{k=0}^{\infty} \sum_{J, J^{\prime}=1}^{\infty} \sum_{\lambda, \lambda^{\prime}=0}^{1}\left(T_{k}^{J \lambda J^{\prime} \lambda^{\prime}}(e, n) \cdot \mathcal{R}_{k}^{J \lambda J^{\prime} \lambda^{\prime}}(\omega)\right),
$$

where the tensor

$$
T_{k m}^{J \lambda J^{\prime} \lambda^{\prime}}(e, \boldsymbol{n})=4 \pi\left\{\left(e \cdot \boldsymbol{Y}_{J}^{(\lambda)}(\boldsymbol{n})\right) \otimes\left(e^{*} \cdot \boldsymbol{Y}_{J^{\prime}}^{\left(\lambda^{\prime}\right)}(\boldsymbol{n})\right)\right\}_{k m}
$$

completely describes the "photon part" of the cross section. We cannot specify here an explicit form of the atomic parameters ("atomic tensors"), $\mathcal{R}_{k m}^{J \lambda J^{\prime} \lambda}(\omega)$, which are independent of $\boldsymbol{e}$ and $\boldsymbol{n}$, since they are determined by the specific dynamical model used to describe the photoprocess considered. However, we can simplify the photon tensor $T_{k m}^{J \lambda J^{\prime} \lambda^{\prime}}(\boldsymbol{e}, \boldsymbol{n})$, which has a universal form that is independent of any particular dynamical model or of any photoprocess being analyzed. Obviously, this tensor has a cumbersome structure, because it contains electromagnetic multipoles with arbitrary ranks $J$ and $J^{\prime}$.

The tensor $T_{k m}^{J \lambda J^{\prime} \lambda^{\prime}}$ depends on three vectors, $\boldsymbol{n}, \boldsymbol{e}$ and $\boldsymbol{e}^{*}$, and the method of multipole expansions developed in Section 2 may be used straightforwardly for its simplification. However, we first demonstrate how the results for this particular case may also be obtained by an algebraic method (cf. the discussion of equation (11) in [3]). Namely, $T_{k m}^{J \lambda J^{\prime} \prime^{\prime}}$ can be easily calculated in the coordinate frame $K$ with $z$-axis directed along the vector $\boldsymbol{n}$. In this frame the nonzero components of $T_{p m}^{J \lambda J^{\prime} \lambda^{\prime}}$ are

$$
\begin{aligned}
& T_{k, \pm 2}^{J \lambda J^{\prime} \lambda^{\prime}}=\frac{1}{2} C_{J 1 J^{\prime} 1}^{k 2} \Pi_{J J^{\prime}}(\mp 1)^{\lambda+\lambda^{\prime}}( \pm 1)^{J+J^{\prime}+k} e_{ \pm 1} e_{ \pm 1}^{*}, \\
& T_{k 0}^{J \lambda J^{\prime} \lambda^{\prime}}=\frac{1}{2} C_{J 1 J^{\prime}-1}^{k 0} \Pi_{J J^{\prime}}(-1)^{\lambda+1}\left(e_{1} e_{-1}^{*}+(-1)^{J+J+\lambda+\lambda^{\prime}+k} e_{-1} e_{1}^{*}\right),
\end{aligned}
$$


where $\Pi_{J J^{\prime}}=\left((2 J+1)\left(2 J^{\prime}+1\right)\right)^{1 / 2}$; in obtaining (34) we used the fact that $e_{0}=0$, because the vector $\boldsymbol{e}$ lies in the $x y$-plane. Now, we have to construct the "minimal" tensor, whose components calculated in the frame $K$ must be equal to those given by equations (34). This tensor should be composed from the vectors involved in the problem, i.e. $\boldsymbol{e}$ and $\boldsymbol{n}$. Let us introduce two orthogonal vectors, $\boldsymbol{e}^{ \pm}=(\boldsymbol{e} \pm \mathrm{i}[\boldsymbol{e} \times \boldsymbol{n}]) / 2$. In the $K$-frame the vector $\boldsymbol{e}^{+}\left(\right.$or $\left.\boldsymbol{e}^{-}\right)$has only one nonzero component $\left(\boldsymbol{e}^{+}\right)_{1}=e_{1}$ (or $\left.\left(\boldsymbol{e}^{-}\right)_{-1}=e_{-1}\right)$. Further, we consider the minimal tensor $\left\{\left\{\boldsymbol{e}^{+} \otimes \boldsymbol{e}^{+}\right\}_{2} \otimes \tilde{Y}_{k-2}(\boldsymbol{n})\right\}_{k m}$. It is easy to verify that in the $K$-frame this tensor has only one nonzero component,

$$
\left\{\left\{\boldsymbol{e}^{+} \otimes e^{+}\right\}_{2} \otimes \tilde{Y}_{k-2}(\boldsymbol{n})\right\}_{k m}=C_{22 k-20}^{k 2} e_{1} e_{1} \delta_{m, 2} .
$$

Thus its component coincides (up to an inessential numerical coefficient) with the component $T_{k 2}^{J \lambda J^{\prime} \lambda^{\prime}}$ given in (34). Similarly, the minimal tensors, corresponding to other components of $T_{k m}^{J \lambda J^{\prime}} \lambda^{\prime}$, may be constructed. After some algebra involving tensor products of $\boldsymbol{e}^{ \pm}$, we arrive at the representation of the photon tensor $T_{k m}^{J \lambda J^{\prime} \lambda^{\prime}}(\boldsymbol{e}, \boldsymbol{n})$ in the form

$$
\begin{aligned}
T_{k m}^{J \lambda J^{\prime} \lambda^{\prime}}(\boldsymbol{e}, \boldsymbol{n}) & =\left[A_{k}^{(0)}-\xi A_{k}^{(1)}\right] \tilde{Y}_{k m}(\boldsymbol{n}) \\
& -A_{k}^{(2)} \sqrt{(2 k-1)(2 k-3)}\left\{\left\{\boldsymbol{e} \otimes e^{*}\right\}_{2} \otimes \tilde{Y}_{k-2}(\boldsymbol{n})\right\}_{k m} \\
& +2 \mathrm{i} A_{k}^{(3)} \sqrt{(2 k-1)(2 k-3)}\left[\left\{\left\{[\boldsymbol{e} \times \boldsymbol{n}] \otimes \boldsymbol{e}^{*}\right\}_{2} \otimes \tilde{Y}_{k-2}(\boldsymbol{n})\right\}_{k m}\right. \\
& \left.+\left\{\left\{\left[\boldsymbol{e}^{*} \times \boldsymbol{n}\right] \otimes \boldsymbol{e}\right\}_{2} \otimes \tilde{Y}_{k-2}(\boldsymbol{n})\right\}_{k m}\right]
\end{aligned}
$$

where $\xi$ is the circular polarization degree of the photon, $\xi=\mathrm{i} \boldsymbol{n} \cdot\left[\boldsymbol{e} \times \boldsymbol{e}^{*}\right]$ (see [2]); the numerical coefficients $A_{k}^{(i)}$ (which depend on $J, J^{\prime}, \lambda$, and $\lambda^{\prime}$ ) may be written as simple combinations of Clebsch-Gordan coefficients:

$$
\begin{aligned}
& A_{k}^{(0)}=\frac{1+(-1)^{\phi}}{4}(-1)^{\lambda} \Pi_{J J^{\prime}}\left[C_{J 1 J^{\prime}-1}^{k 0}+(-1)^{\lambda^{\prime}} \sqrt{\frac{k(k-1)}{(k+1)(k+2)}} C_{J 1 J^{\prime} 1}^{k 2}\right], \\
& A_{k}^{(1)}=\frac{1-(-1)^{\phi}}{4}(-1)^{\lambda} \Pi_{J J^{\prime}} C_{J 1}^{k 0} J_{J^{\prime}-1}, \\
& A_{k}^{(2)}=\frac{1+(-1)^{\phi}}{2} \frac{(-1)^{\lambda+\lambda^{\prime}}}{\sqrt{(k+1)(k+2)}} \Pi_{J J^{\prime}} C_{J 1}^{k 2} J_{J^{\prime} 1}, \\
& A_{k}^{(3)}=\frac{1-(-1)^{\phi}}{4} \frac{(-1)^{\lambda+\lambda^{\prime}}}{\sqrt{(k+1)(k+2)}} \Pi_{J J^{\prime}} C_{J 1 J^{\prime} 1}^{k 2},
\end{aligned}
$$

where $\phi=J+J^{\prime}+\lambda+\lambda^{\prime}+k$. Thus, in (36) the tensor $T_{k m}^{J \lambda J^{\prime} \lambda^{\prime}}(\boldsymbol{e}, \boldsymbol{n})$ is presented as the sum of three simple tensors of rank $k$ : one of them is a spherical harmonic and the other two are simple tripolar harmonics in which two of the internal tensors are rank-1 tensors (vectors $\boldsymbol{e}$ and $\boldsymbol{e}^{*}$ ). In other words, the result (36) gives the multipole expansion of the tensor $T_{k m}^{J \lambda J^{\prime} \lambda^{\prime}}(\boldsymbol{e}, \boldsymbol{n})$ in the terminology of section 2.

As noted above, the tensor $T_{k m}^{J \lambda J^{\prime} \lambda^{\prime}}$ may also be obtained by the regular method presented in section 2, which is based on the general multipole expansion (8). Using $T_{k m}^{J \lambda J^{\prime} \lambda}\left(\boldsymbol{e}, \hat{r}^{\prime}\right)$ from (33) instead of $T_{j m}\left(\boldsymbol{r}^{\prime}\right)$ in (9) and taking into account known relations between $\boldsymbol{Y}_{J}^{(\lambda)}(\hat{r})$ and the spherical harmonics $Y_{l m}(\hat{r})$ [9], one obtains after a number of manipulations the differential form of the photon tensor:

$$
\begin{aligned}
T_{k m}^{J \lambda J^{\prime} \lambda^{\prime}}(e, \boldsymbol{n})= & {\left[A_{k}^{(0)}-\xi A_{k}^{(1)}-A_{k}^{(2)} \frac{|e \cdot \nabla|^{2}}{\sqrt{k(k-1)}}\right.} \\
& \left.+A_{k}^{(3)} \frac{4 \mathrm{i}}{\sqrt{k(k-1)}} \operatorname{Re}\left\{\left(e^{*} \cdot \nabla\right)(e \cdot[\boldsymbol{n} \times \nabla])\right\}\right]\left.r^{k} \tilde{Y}_{k m}(\hat{r})\right|_{r=n}
\end{aligned}
$$


Note that the differentiation in this equation may be performed straightforwardly, using the invariant differentiation technique (see [3] and especially the appendix therein). Then the result coincides with that in (36). Nevertheless, the differential form (38) may be the most convenient one for concrete applications, since here the whole dependence on the tensor projection $m$ is concentrated in only one spherical harmonic, $Y_{k m}(\hat{r})$. Thus, it simplifies the calculation of the scalar product of the photon tensor with the "atomic tensors" $\mathcal{R}_{k m}^{J \lambda J^{\prime} \lambda^{\prime}}$ in equation (32) for the cross sections. After calculation of this scalar product, the result of vector differentiation may be presented directly in terms of those physical vectors that are involved in the particular expression for the "atomic tensors" $\mathcal{R}_{k m}^{J \lambda J^{\prime} \lambda^{\prime}}(\omega)$ in (32).

\subsection{Discussion}

Obviously, the parametrization of a photoprocess cross section by equations (32) and (36) or (38) is very general. It simplifies considerably in a number of special cases, depending on the particular photoprocess, the symmetry of the target atom, etc. Specifically, the structure of the atomic tensor, $\mathcal{R}_{k m}^{J \lambda J^{\prime} \lambda^{\prime}}(\omega)$, in (32) is determined by the vector physical variables of the problem other than $\boldsymbol{e}$ and $\boldsymbol{n}$, for example by the spins and the momenta of the incident and/or escaping electrons, by the polarization state of the target, etc. For instance, for the case of emission or absorption of a single photon by an unpolarized target without observation of the target polarization in the final state there are no other vectors in the problem besides $\boldsymbol{e}$ and $\boldsymbol{n}$. Thus, only partial cross sections $\mathcal{R}_{k m}$ with $k=0$ (scalars) in (32) are nonzero. If only one vector, say $\boldsymbol{b}$, is inherent to the "atomic part" of the cross section (e.g. the electron momentum $\boldsymbol{p}$, or the angular momentum $\boldsymbol{j}$ of a polarized target), then $\mathcal{R}_{k m}^{J \lambda J^{\prime} \lambda^{\prime}}$ will be proportional to $\{\boldsymbol{b}\}_{k m}$, i.e. to the spherical harmonic of $\boldsymbol{b}$. Moreover, after the contraction of this spherical harmonic with those involved in equations (36) or (38) the final result will be expressed in terms of Legendre polynomials (which depend on the angle between $\boldsymbol{b}$ and $\boldsymbol{n}$ ) whose number is finite (for $\boldsymbol{b}=\boldsymbol{j}$ ) or infinite (for $\boldsymbol{b}=\boldsymbol{p}$ ).

Note that $T_{k m}^{J \lambda J^{\prime} \lambda^{\prime}}$ reduces to $\left\{\boldsymbol{e} \otimes \boldsymbol{e}^{*}\right\}_{k m}$ in the electric dipole approximation, when $J$ $=J^{\prime}=\lambda=\lambda^{\prime}=1$. For this case, the cross section of any photoprocess reduces to the simple form,

$$
\frac{\mathrm{d} \sigma}{\mathrm{d} \Omega}=\sum_{k=0}^{2}\left(\left\{e \otimes e^{*}\right\}_{k} \cdot \mathcal{R}_{k}^{\mathrm{dip}}(\omega)\right),
$$

where the atomic tensor $\mathcal{R}_{k m}^{\text {dip }}(\omega)$ does not depend on $\boldsymbol{k}$ and $\boldsymbol{e}$. Properties of the photon polarization tensor $\left\{\boldsymbol{e} \otimes \boldsymbol{e}^{*}\right\}_{k}$ and its various parametrizations were discussed in [3,4]. We also presented in these works convenient parametrizations of the cross section (39) taking into account the explicit forms of the tensors $\mathcal{R}_{k m}{ }^{\mathrm{dip}}(\omega)$ for a number of the most important photoprocesses: the angular distribution and polarization effects in photon emission by a polarized target, the angular distribution of polarized electrons in photoionization of polarized atoms having an arbitrary angular momentum, and also the photon polarization effects in electron bremsstrahlung and in two-electron photoionization from unpolarized targets. In all these cases, the electric dipole approximation was used for the photon-target interaction. The analysis of the general structure of the photon tensor $T_{k m}^{J \lambda J^{\prime} \lambda^{\prime}}$ presented above allows one to extend these results to account accurately for non-dipole (or retardation) effects in electron-photon interactions.

The detailed analyses for concrete photoprocesses will be published elsewhere. We discuss here only some general features of equations (36) and (38). Firstly, the term with $A_{k}^{(3)}$ in (36) vanishes in the electric dipole approximation, since it involves explicitly the wavevector $\boldsymbol{k}=(\omega / c) \boldsymbol{n}$, which contributes only to beyond-electric-dipole-approximation 
results. Moreover, this term vanishes also for completely circularly polarized photons (with $\xi= \pm 1$ ), in which case the general result for $T_{k m}^{J \lambda J^{\prime} \lambda^{\prime}}$ reduces to the following:

$$
T_{k m}^{J \lambda J^{\prime} \lambda^{\prime}}(\boldsymbol{e}, \boldsymbol{n})=\left[A_{k}^{(0)}-\xi A_{k}^{(1)}+A_{k}^{(2)} \frac{1}{2} \sqrt{k(k-1)}\right] \tilde{Y}_{k m}(\boldsymbol{n}) .
$$

(For the case of unpolarized photons $(\xi=0)$ the term with $A_{k}^{(1)}$ should be omitted from this equation.) Generally, if a term with $\xi$ appears in a cross section it describes the circular dichroism (CD) effect, i.e. the difference in cross sections for right and left helicities of an (in general) elliptically polarized photon. CD is typical for photoprocesses with polarized targets and/or electrons, although in some cases it appears also in photoprocesses with unpolarized particles (see, e.g., $[3,19])$. Thus, the results presented in this paper may be useful for the rigorous analysis of the contribution of non-dipole corrections to dichroism effects for VUV- and x-ray photons. In particular, recently it has been demonstrated that in VUV photoionization processes the non-dipole effects may make sizable contributions both to the angular distribution of photoelectrons from polarized atoms [20] and to the spin polarization of photoelectrons ejected from unpolarized targets [21].

\section{Conclusion}

In this paper we have presented a method which may be useful for the general problem of separating kinematical and dynamical factors in atomic processes as well as for other atomic and molecular problems dealing with a number of vector quantities. In the usual case, the use of standard methods of angular momentum algebra results in these vectors entering the final results for cross sections through complicated tensor constructions $T_{j m}\left(\boldsymbol{a}_{1}, \boldsymbol{a}_{2}, \ldots\right)$. In order to extract the dependence of $T_{j m}$ on the vector directions in an explicit form, we offer a method for obtaining multipole expansions of irreducible tensor sets $T_{j m}\left(\boldsymbol{a}_{1}, \boldsymbol{a}_{2}, \ldots\right)$ in terms of multipolar harmonics dependent on the directions of $\boldsymbol{a}_{\boldsymbol{i}}$. The formal multipole expansion of an irreducible tensor in a symbolic differential form is obtained in Section 2. The coefficients of this expansion are obtained either by means of invariant vector differentiation or by selecting a suitable coordinate frame. Some details of such calculations are illustrated in Section 3 for the example of the multipole expansion of two-center tensor functions dependent on the distance between two points specified by the vectors $\boldsymbol{r}_{1}$ and $\boldsymbol{r}_{2}$. (For another illustration, see also the appendix for higher-order "gradient formulae" for spherical harmonics.) In Section 4 we derived the regular multipole expansion (in terms of bipolar harmonics) of the invariant vector representation of FRM. For a special choice of rotation parameters, this result reduces to the expansion of spherical harmonics in terms of Legendre polynomials. In order to illustrate the application of our invariant techniques to concrete physical problems, in Section 5 we employed this technique for the reduction of tensor products of electric and magnetic multipoles, which appear, in particular, in the analysis of photoprocesses beyond the electric dipole approximation. These results thus provide a basis for analyzing non-dipole effects in the polarization and angular dependences of photoprocesses in the VUV- and x-ray regions. We note finally that irreducible tensor sets $T_{j m}$, and in particular their most important concrete examples, the FRM $R_{k m}^{j}(\Omega)$ and the spherical harmonics $Y_{j m}(\boldsymbol{n})$, are not only fundamental objects of the quantum theory of angular momentum but also have an interdisciplinary interest. Thus any new results for these objects, such as those presented here, have an evident importance and potential usefulness in various applications. 


\section{Acknowledgments}

This work has been supported in part by NSF Grant PHY-0070980, by RFBR Grant 0002-17843 and by the grant program "Universities of Russia."

\section{Appendix. Higher-order "gradient formulae" for spherical harmonics}

In order to obtain the "generalized gradient formula" (14) we start with the following operator identity:

$\left\{\{\nabla\}_{l} \otimes f(r) \tilde{Y}_{j}(\hat{r})\right\}_{l^{\prime} m^{\prime}}=\sum_{k=0}^{l}\left(\begin{array}{l}l \\ k\end{array}\right)\left[\hat{\mathrm{d}}^{l-k} \frac{f(r)}{r^{j}}\right]\left\{\left\{\{r\}_{l-k} \otimes\{\nabla\}_{k}\right\}_{l} \otimes r^{j} \tilde{Y}_{j}(\hat{r})\right\}_{l^{\prime} m^{\prime}}$,

where

$$
\hat{\mathrm{d}} \equiv \frac{1}{r} \frac{\mathrm{d}}{\mathrm{d} r}
$$

This identity may be verified from the standard Leibniz differentiation rule when the tensors are expressed in terms of their components. In our derivation, however, we employed the auxiliary relation,

$$
\{\nabla\}_{k q} \varphi(r)=\{r\}_{k q} \hat{\mathrm{d}}^{k} \varphi(r),
$$

which can be verified straightforwardly for an arbitrary function $\varphi(r)$. For the calculation of the tensor product on the rhs of (A.1) we employ the rank-decreasing operator for the spherical harmonic, defined by equation (A.7) of [3]:

$$
\begin{aligned}
& \hat{\mathcal{O}}_{k q}^{j-}(r, \nabla)=(-1)^{k} \sqrt{\frac{2^{k}(2 j-2 k+1) !}{(2 j+1) !}} \frac{1}{r^{j-k}}\{\nabla\}_{k q} r^{j}, \\
& \left\{\hat{\mathcal{O}}_{k}^{j-}(r, \nabla) \otimes \tilde{Y}_{j}(\hat{r})\right\}_{a \alpha}=\delta_{a, j-k} \tilde{Y}_{j-k, \alpha}(\hat{r}) .
\end{aligned}
$$

After rewriting the tensor product of gradient-operators in (A.1) in terms of the rank-decreasing operators (A.3), we use the tensor recoupling rule [9] and equation (A.4) to obtain the following result:

$$
\begin{aligned}
\left\{\left\{\{\boldsymbol{r}\}_{l-k} \otimes \hat{\mathcal{O}}_{k}^{j-}(r, \nabla)\right\}_{l} \otimes \tilde{Y}_{j}(\hat{r})\right\}_{l^{\prime} m^{\prime}}=(-1)^{j+l+l^{\prime}} \sqrt{(2 l+1)(2 j-2 k+1)} \\
\times\left\{\begin{array}{ccc}
l-k & k & l \\
j & l^{\prime} & j-k
\end{array}\right\}\left\{\{r\}_{l-k} \otimes \tilde{Y}_{j-k}(\hat{r})\right\}_{l^{\prime} m^{\prime}} .
\end{aligned}
$$

Substituting into (A.5) the auxiliary relation

$$
\left\{\{r\}_{l-k} \otimes \tilde{Y}_{j-k}(\hat{r})\right\}_{l^{\prime} m^{\prime}}=\sqrt{\frac{(l-k) !}{(2 l-2 k-1) ! !}} C_{l-k 0 j-k 0}^{l^{\prime} 0} r^{l-k} \tilde{Y}_{l^{\prime} m^{\prime}}(\hat{r}),
$$

which follows from the definition of the spherical harmonics given in (5), the spherical harmonic $\tilde{Y}_{l^{\prime}}(\hat{r})$ can now be removed from the sum over $k$ in (A.1). Calculating explicitly the $6 j$-symbol in (A.5) together with the Clebsch-Gordan coefficient in (A.6), after some tedious algebra we obtain the compact operator expression (14) for the tensor construction on the rhs of equation (13).

The key element in the derivation of (14) was the use of the rank-decreasing operators for spherical harmonics, $\hat{\mathcal{O}}_{k}^{j-}(r, \nabla)$. Obviously, similar calculations may also be performed using the rank-increasing operators, $\hat{\mathcal{O}}_{k}^{j+}(r, \nabla)$, defined in [3]. In this case we obtain instead of (14)

$\left\{\{\nabla\}_{l} \otimes f(r) \tilde{Y}_{j}(\hat{r})\right\}_{l^{\prime} m^{\prime}}=\sqrt{\frac{l !}{(2 l-1) ! !}} C_{l 0 j 0}^{l^{\prime} 0} r^{l^{\prime}} \tilde{Y}_{l^{\prime} m^{\prime}}(\hat{r}) \hat{\mathrm{d}}^{g-j} \frac{1}{r^{2 g-2 l+1}} \hat{\mathrm{d}}^{g-l^{\prime}} r^{j+1} f(r)$. 
Comparing (A.7) and (14), we obtain the nontrivial operator identity

$$
r^{l^{\prime}} \hat{\mathrm{d}}^{g-j} \frac{1}{r^{2 g-2 l+1}} \hat{\mathrm{d}}^{g-l^{\prime}} r^{j+1} f(r)=\frac{1}{r^{l^{\prime}+1}} \hat{\mathrm{d}}^{g-l^{\prime}} r^{2 g+1} \hat{\mathrm{d}}^{g-j} \frac{f(r)}{r^{j}},
$$

which is valid for any analytical function $f(r)$. This identity can be verified also straightforwardly for the special function $f(r)=r^{n}$, making use of the relation $\hat{\mathrm{d}}^{k} r^{\alpha}=(-2)^{k} r^{\alpha-2 k}$ $(-\alpha / 2)_{k}$, where $(\alpha)_{k}=\Gamma(\alpha+k) / \Gamma(\alpha)$ is the Pochhammer symbol. Thus, since the function $f(r)$ can be expanded in a power series, the identity (A.8) is correct for an arbitrary function $f(r)$ as well.

\section{References}

[1] Jacob M and Wick G C 1959 Ann. Phys., NY 7404

[2] Berestetskii V B, Lifshitz E M and Pitaevskii L P 1982 Quantum Electrodynamics (Oxford: Pergamon)

[3] Manakov N L, Marmo S I and Meremianin A V 1996 J. Phys. B: At. Mol. Opt. Phys. 292711

[4] Manakov N L, Meremianin A V and Starace A F 1998 Phys. Rev. A 573233

[5] Manakov N L, Meremianin A V and Starace A F 2000 Phys. Rev. A 61022103

[6] Manakov N L, Meremianin A V and Starace A F 2001 Phys. Rev. A 64032105

[7] Manakov N L and Meremianin A V 1997 Zh. Eksp. Teor. Fiz. 1111984 (Engl. transl. 1997 Sov. Phys.-JETP 84 1080)

[8] Fano U and Racah G 1959 Irreducible Tensorial Sets (New York: Academic)

[9] VarshalovichDA,MoskalevANand KhersonskiiVK1988 Quantum Theory of Angular Momentum (Singapore: World Scientific)

[10] Taichenachev A V, Tumaikin A V and Yudin V I 2000 Zh. Eksp. Teor. Fiz. 11877 (Engl. transl. 2000 Sov. Phys.-JETP 91 67)

[11] Bezverbnyi A V 2000 Zh. Eksp. Teor. Fiz. 1181066 (Engl. transl. 2000 Sov. Phys.-JETP 91 921)

[12] Manakov N L, Meremianin A V, Maquet A and Carney J P J 2000 J. Phys. B: At. Mol. Opt. Phys. 334425

[13] Sack R A 1964 J. Math. Phys. 5252

[14] Avery J 1979 Int. J. Quantum. Chem. 161265

[15] Wen Z Y and Avery J 1985 J. Math. Phys. 26396

[16] Rose M E 1955 Multipole Fields (New York: Wiley)

[17] Happer W 1968 Ann. Phys., NY 48579

[18] Erdelyi A, Magnus W, Oberhettinger F and Tricomi F G 1955 Higher Transcendental Functions (Bateman Manuscript Project) vol 2 (New York: McGraw-Hill)

[19] Briggs J S and Schmidt V 2000 J. Phys. B: At. Mol. Opt. Phys. 33 R1

[20] Grum-Grzhimailo A N 2001 J. Phys. B: At. Mol. Opt. Phys. 34 L359

[21] Cherepkov N A and Semenov S K 2001 J. Phys. B: At. Mol. Opt. Phys. 34 L211 